\title{
Staphylococcus aureus Small Colony Variants (SCVs): a road map for the metabolic pathways involved in persistent infections
}

\section{Richard A. Proctor ${ }^{1 *}$, André Kriegeskorte ${ }^{2}$, Barbara C. Kahl ${ }^{2}$, Karsten Becker ${ }^{2}$, Bettina Löffler ${ }^{2}$ and Georg Peters ${ }^{2}$}

${ }^{1}$ Departments of Medical Microbiology/Immunology and Medicine, University of Wisconsin School of Medicine and Public Health, Madison, WI, USA

2 Institute of Medical Microbiology, University Hospital, Münster, Münster, Germany

\section{Edited by:}

Wolfgang Eisenreich, Technische Universität München, Germany

\section{Reviewed by:}

Martin I. Voskuil, University of Colorado Denver, USA

Alain Charbit, University Paris

Descartes, France

\section{*Correspondence:}

Richard A. Proctor, Departments of Medical Microbiology/Immunology and Medicine, University of Wisconsin School of Medicine and Public Health, 835 Asa Gray, Ann

Arbor, MI 48105-2565, USA

e-mail: rap@wisc.edu
Persistent and relapsing infections, despite apparently adequate antibiotic therapy, occur frequently with many pathogens, but it is an especially prominent problem with Staphylococcus aureus infections. For the purposes of this review, persistence will encompass both of the concepts of long term survival within the host, including colonization, and the concept of resisting antibiotic therapy even when susceptible in the clinical microbiology laboratory. Over the past two decades, the mechanisms whereby bacteria achieve persistence are slowly being unraveled. S. aureus small colony variants (SCVs) are linked to chronic, recurrent, and antibiotic-resistant infections, and the study of SCVs has contributed significantly to understanding of persistence. In our earlier work, defects in electron transport and thymidylate biosynthesis were linked to the development of the SCV phenotype (reviewed in 2006), thus this work will be discussed only briefly. Since 2006, it has been found that persistent organisms including SCVs are part of the normal life cycle of bacteria, and often they arise in response to harsh conditions, e.g., antibiotics, starvation, host cationic peptides. Many of the changes found in these early SCVs have provided a map for the discovery mechanisms (pathways) for the development of persistent organisms. For example, changes in RNA processing, stringent response, toxin-antitoxin, ribosome protein L6 (RplF), and cold shock protein B (CspB) found in SCVs are also found in other persisters. In addition, many classic persister organisms also show slow growth, hence SCVs. Recent work on S. aureus USA300 has elucidated the impact of aerobic expression of arginine deiminase genes on its ability to chronically colonize the skin and survive in abscesses. S. aureus SCVs also express arginine deiminase genes aerobically as well. Thus, many pathways found activated in electron transport type of SCVs are also increased in persisters that have intact electron transport. Many of these changes in metabolism result in slow growth; hence, small colonies are formed. Another common theme is that slow growth is also associated with reduced expression of virulence factors and enhanced uptake/survival within host cells. These adaptations to survive within the host are rooted in responses that were required for organisms to survive in a harsh environment long before they were mammals on the earth.

Keywords: Staphylococcus aureus, small colony variants, metabolism, RNA processing, post-transcriptional, persistence

\section{INTRODUCTION}

Persistent and relapsing infections, despite organism susceptibility and apparently adequate antibiotic therapy, occur frequently with many pathogens, but it is an especially prominent problem with Staphylococcus aureus infections (Lowy, 1998). The basis for persistence has been slowly unraveled over the past two decades, and many of the pathways involve changes in metabolism. One phenotype of microorganisms has helped to pave the way to understand persistence, and this is represented by small colony variants (SCVs) (Proctor et al., 2006). The linkage between S. aureus SCVs and persistent infection was first reported in a small clinical series in 1995 (Proctor et al., 1995). Because S. aureus SCVs were able to establish an intracellular infection in cultured cells, it was hypothesized that this might form the basis for the development of persistent infections (Balwit et al., 1994). Moreover, the instability of these SCVs wherein they could revert to the parental normal phenotype would also provide a mechanism for relapsing, virulent infections. An important part of the ability to persist was associated with the quiescent metabolic state (Proctor et al., 1995). S. aureus SCVs were found to produce fewer lytic enzymes, thereby allowing them to persist within the host cells (Proctor et al., 2006). Since then, further work 
has demonstrated enhanced uptake by $S$. aureus SCVs due to a high expression of adhesins that facilitate host cell uptake (Sendi and Proctor, 2009; Tuchscherr et al., 2010). Early studies suggested that the intracellular milieu could select for SCVs (Vesga et al., 1996), perhaps because cationic antimicrobial peptides, e.g., lactoferrin, provide the selective pressure within host cells (Samuelsen et al., 2005; Gläser et al., 2014). While early studies suggested that the intracellular milieu could select for SCVs (Vesga et al., 1996), more recent and detailed studies demonstrated that a high percentage of the initial inoculum (as much as $25 \%$ ) of $S$. aureus could undergo phenotypic switching to produce SCVs (Tuchscherr et al., 2011). This was shown to not only occur in tissue culture, but also within the kidneys and bones of these intravenously challenged mice (Horst et al., 2012). The SCVs that persisted stimulated a reduced immune response, expressed increased adhesins, and reduced toxins (Tuchscherr et al., 2010). Moreover, the failure of $S$. aureus SCVs to stimulate host cells to produce hypoxia-inducible factor, which would normally alert the host to the presence of intracellular pathogens, also is important for promoting persistence (Werth et al., 2010). Finally, persistence of $S$. aureus SCVs may also relate to the relative inefficiency of antibiotics to clear the organisms with host cells (Garcia et al., 2013). Thus, S. aureus SCVs have been established as intracellular pathogens that persist within the host. This review will largely concentrate upon information that has been published since the 2006 review of SCVs (Proctor et al., 2006) and will emphasize metabolic mechanisms involved in persistence. S. aureus SCVs as a model persister organism

How do $S$, aureus SCVs relate to the broader concept of persistence? Many other species of bacteria that persist within the host also form SCVs (Proctor et al., 2006). Hence, the specific example of $S$. aureus SCVs is also found within many other pathogenic species. In addition, persisters share many characteristics, and these are also part of the SCV life cycle. Persisters and S. aureus SCVs grow slowly due to decreased metabolic activity (Amato et al., 2014). A subset of organisms within many bacterial populations can be part of the normal growth cycle (Lechner et al., 2012), and this is certainly true for S. aureus SCVs, which are part of the normal growth cycle that occurs without any external stresses (Massey et al., 2001; Edwards, 2012). However, persisters also form as a response to harsh conditions such as antibiotics, acid, and starvation stresses (Morikawa et al., 2010). Biofilm formation can be thought of as a persister strategy (Abdallah et al., 2014). Naturally, persistence is inter-related to chronic colonization and chronic infections as these may be considered types of persistence.

\section{ELECTRON TRANSPORT TYPE OF S. AUREUS SCVS}

The initial description of $S$. aureus SCV persisters identified alterations in electron transport due to mutations in hemin and menadione biosynthetic genes, which resulted in a loss of menaquinone and the heme prosthetic group in cytochromes (Proctor et al., 2006). Others found that mutations in the cytochrome c assembly protein (CtaA) also produced SCVs (Clements et al., 1999). Thymidine auxotrophic SCVs were soon added (Proctor et al., 2006) to the list of metabolic changes in SCVs. Mutations in thyA were found to produce SCVs that were phenotypically very similar to the electron transport deficient (Chatterjee et al., 2008). The reason that thymidine-dependent SCVs behaved like electron transport defective organisms came through decreased Krebs cycle activity (Chatterjee et al., 2005, 2007). Of course, when Krebs cycle is decreased, electron transport is down regulated. It was found that thy $A$ mutation was associated with decreased aconitase (CitB) abundance. A further connection became apparent wherein $\mathrm{Cit} B$ expression requires ClpC, which is reduced in thy A mutants (Chatterjee et al., 2005, 2007, 2008). Hence, thy $A$ mutants are grouped with organisms defective in electron transport. All of the electron transport types of SCVs are able to persist within the host and/or cultured mammalian cells.

\section{CHANGES IN RNAIII METABOLISM IN ELECTRON TRANSPORT GROUP OF SCVs}

As noted above, slow growth is found in many, but not all, organisms that persist. When examining clinical and tissue-cultured induced S. aureus SCVs, only $\sim 20 \%$ can be assigned to a defined auxotrophy (Vesga et al., 1996; Proctor et al., 2006; Tuchscherr et al., 2011). Therefore, the underlying mechanism for forming a persistent SCV is unknown. Publications in the past six years have revealed a number of mutations that result in SCVs, but most of these have not been examined in clinical SCVs. Surprisingly, many of these newer mutations can be grouped as being involved with the ribosome and RNA processing. This will be discussed in the balance of this article.

One of the first indications that electron transport type of $S$. aureus SCVs were atypical for RNA processing came from the observation that there was a complete absence of RNAIII (Vaudaux et al., 2002; Kohler et al., 2003, 2008; Kahl et al., 2005), which is the effector molecule in the quorum sensing apparatus, agr (accessory gene regulator), in S. aureus (Novick and Geisinger, 2008). RNAIII arises from nontranslated portion of the hld (hemolysin D) gene in the agr operon. RNAIII positively regulates production of toxins and proteases but negatively regulates adhesins.

Because $a g r$ is a quorum-sensing system, one might postulate that the decreased growth of electron-transport types of SCVs simply do not reach sufficient density to activate the quorumsensing pathway. This seems unlikely for several reasons. The first is that SCV colonies on blood agar, wherein the local density is very high, fail to produce hemolytic colonies, and the hemolysins are controlled by agr/RNAIII (Proctor et al., 2006). The second is that RNAIII is not found in any detectable amount in SCVs (Vaudaux et al., 2002; Kohler et al., 2003, 2008; Kahl et al., 2005), which is distinctly unusual in that a basal level of RNAIII is produced in $S$. aureus during all phases of growth (Novick and Geisinger, 2008). Surprisingly, mutation in citB produces a major growth defect, yet a 14-fold increase in RNAIII was found in the citB mutant as compared to the parent strain $S$. aureus strain. This work was performed in a clinical isolate, SA564, in TSB supplemented with $0.25 \%$ glucose (Somerville et al., 2002). The differences in RNAIII production were found in the postexponential phase of growth, with no differences in the exponential phase of growth. Finally, RNAIII is reduced under anaerobic conditions in S. aureus MN8 along with the production of toxic 
shock syndrome toxin-1 (TSST-1) (Pragman et al., 2004), which is regulated by RNAIII. However, the anaerobic suppression of TSST-1 production can be reversed if excess quantities of pyruvate are added to the growth medium, resulting in TSST-1 production during late stationary phase (Table 1 ). This shows that the anaerobic conditions alone do not block RNAIII-regulated production of TSST-1, which is consistent with an earlier report where both pyruvate and uracil were added to the culture medium (Sarafian and Morse, 1987). Taken together, these data strongly suggest an altered RNAIII metabolism when electron transport is reduced, which is not simply due to reduced growth/concentration of the quorum-sensing peptide.

There is a strong inverse connection between $a g r$, hence RNAIII, and persistent infection. The loss of the major virulence factor regulator in $S$. aureus, $a g r$, has been associated with chronic infections. These agr mutants have been obtained from clinical isolates from patients with long-term lung infections (cystic fibrosis) (Kahl et al., 2005; Hirschhausen et al., 2013), chronic catheter infections (Rothfork et al., 2003; Yarwood and Schlievert, 2003), and chronic soft tissue infections (Schwan et al., 2003; Beenken et al., 2004). Of interest, the development of skin abscesses requires the presence of agr to establish the initial infection, but persistence within the abscess is associated with decreased RNAIII (Wright et al., 2005). Thus, these infections are strongly linked to situations wherein the organism persists within the host and resists antibiotic therapy. This suggests that a complete absence of the RNAIII effector molecule from the agr operon is associated with persistence.

\section{THE RNA DEGRASOME AND SCVs}

Of interest, small colonies and changes in RNA metabolism are seen in mutants in $c s h A, c s p B, r s a E, r p l F, m a z F$, and $r s h$ (see Table 2). The role of these mutations in persistent infection is discussed in the balance of this manuscript.

Low levels of RNAIII can occur by two mechanisms. The $a g r$ quorum-sensing operon may produce less RNAIII or there may be increased RNAIII degradation to account for low levels of RNAIII. As noted above, the low RNAIII levels in electron transport type of SCVs are more likely related to increased RNAIII degradation. The RNA degrasome in $S$. aureus has recently been elucidated (Roux et al., 2011; Marincola et al., 2012; Redder and Linder, 2012; Oun et al., 2013), and a number of degrasome proteins are increased in SCVs as suggested by transcriptomics and proteomics data from hemB and menD mutant SCVs (Seggewiss

Table 1 | Anaerobic production of toxic shock syndrome toxin - 1 .

\begin{tabular}{lc}
\hline $\mathbf{m M}$ Pyruvate added & ng TSST-1/10 $\mathbf{~ c f u}$ \\
\hline 0 & 0.7 \\
40 & 0.05 \\
200 & 24.6 \\
400 & 63
\end{tabular}

Exogenous pyruvate and anaerobic toxin production in S. aureus MN8 grown in tryptic soy broth for $18 \mathrm{~h}$ under anaerobic conditions, and supernatants collected for TSST-1 measurements. TSST-1 was measured by an ELISA as previously described (McNamara et al., 2009). et al., 2006; Kriegeskorte et al., 2011). A model of the RNA degrasome is provided in Figure 1.

The hemB mutant SCV has increased enolase, helicase (CshA), RsaA (a small noncoding RNA involved in stress responses and biofilm formation), $\operatorname{csp} B$ (cold shock protein B), SigB, SarA, and FruR (Seggewiss et al., 2006; Kriegeskorte et al., 2011). Of note, metabolism is embedded in the degrasome with enolase and phosphofructokinase (Pfk) directly interacting with CshA, but the effects that they have on CshA has yet to be characterized. Some of the targets of the degrasome are small nonprotein coding RNAs (sRNAs) that control central carbon metabolism (Geissmann et al., 2009; Bohn et al., 2010; Felden et al., 2011; Guillet et al., 2013; Romilly et al., 2014; Xue et al., 2014), and SCVs show changes in the levels of these sRNAs (Abu-Qatousch et al., 2010). This is not surprising as SCVs showed markedly increased levels of SigB (Mitchell et al., 2013), which positively regulates RsaA (Felden et al., 2011), and decreased levels of RsaE that regulates central carbon metabolism. The low levels of RsaE in SCVs are likely due to low levels of RNAIII, which is positively RsaE (Donegan and Cheung, 2009; Donegan et al., 2010; Guillet et al., 2013). In Bacillus subtilis, CspB associates with CshA (Hunger et al., 2006). CspB is included in the degrasome, and it is increased in hemB mutant SCVs (Seggewiss et al., 2006; Kriegeskorte et al., 2011). RNase Y processes saeRS mRNA so that it is active. (Marincola et al., 2012). Thus, as changes in RNA processing impact virulence factor production, TCA cycle, and biofilms, a number of the phenotypic characteristics of SCVs may be attributable to RNA processing.

A mutation in the ATP-dependent hellicase, $c \operatorname{sh} A$, in $S$. aureus results in a temperature-dependent $\mathrm{SCV}$, with reduced growth at $30^{\circ} \mathrm{C}$ and complete growth inhibition at $22^{\circ} \mathrm{C}$ (Oun et al., 2013). Disruption of $c \operatorname{sh} A$ results in higher RNAIII stability with increased hemolysis and reduced biofilm formation. Knocking agr $A$ reversed the phenotype, indicating that $\operatorname{csh} A$ is genetically upstream of $\operatorname{agr}$ (Oun et al., 2013). In electron transport deficient SCVs, the higher levels of CshA might be expected to have a role in the lower levels of RNAIII.

As noted above, another potential element of the degradosome may be CspB. Of interest, a mutation in $\operatorname{cspB}$ in S. aureus also produces an SCV with reduced pigment production and increased resistance to aminoglycosides as found in electron transport SCVs (Duval et al., 2010). Other characteristics of SCVs such as RNAIII levels and expression of adhesins has not been studied. Similarly, the ability of the $\operatorname{csp} B$ mutant to persist has also not been reported.

\section{SMALL RNAs AND SCVs}

Based on investigations of cDNA libraries, phenotype-specific expression of sRNAs have been described for S. aureus (AbuQatousch et al., 2010). Selected sRNAs have also been found to in the hemB mutant SCV as compared to the wild type strain (Abu-Qatousch et al., 2010). Over expression of RsaE give SCVs that have decreased TCA cycle activity (Geissmann et al., 2009; Bohn et al., 2010). RsaE pairs with mRNAs of citB, citZ, and sucCD, thereby inhibiting their translation and reducing TCA cycle. While detailed metabolic studies are not available, one would anticipate that these organisms would have reduced electron transport and behave like electron transport deficient 
Table 2 | Comparison of phenotypes in SCVs and persisters.

\begin{tabular}{|c|c|c|c|c|c|c|c|c|}
\hline Phenotype & $\begin{array}{l}\mathrm{SCV} \text { (men/hem/ctaA) } \\
\text { Also, unsatu- } \\
\text { rated fatty acids }\end{array}$ & $\operatorname{SCV}(\operatorname{thy} A)$ & $\Delta c s h A$ & $\Delta \operatorname{csp} B$ & $\begin{array}{l}\text { RsaE over } \\
\text { production }\end{array}$ & $\begin{array}{l}\text { rplF mutant } \\
\text { (Ribosome } \\
\text { protein L6) }\end{array}$ & $\begin{array}{l}\text { Increased } \\
\text { MazF } \\
\text { (Toxin -AT) }\end{array}$ & $\begin{array}{l}\text { Rsh constitutively } \\
\text { active(RelA) }\end{array}$ \\
\hline Colony size & Small & Small & $\operatorname{Small}\left(22^{\circ} \mathrm{C}\right)$ & Small & Small & Small & Small & Small \\
\hline Hla & Decrease & Decrease & Increase & & & & Decreased & Increase \\
\hline HId & Decrease & Decrease & Increase & & & & & Increase \\
\hline Spa & Decrease & Increase & Decrease & & & & Decrease & Decrease \\
\hline FnBP & Increase & Increase & & & & & & Increase \\
\hline RNAIII & Decrease & Decrease & Increase & & Decreased & & & Increase $(\downarrow R n p A)$ \\
\hline CPS & Increase & Decrease & Increase & & & & & Increase \\
\hline Ilv pathway & Increase & & & & & & & Increase \\
\hline ppGpp & & & & & & & & Increase \\
\hline Cell wall thick & Increased & Increased & & & & & & \\
\hline Pigment & Decreased & Decreased & & Decreased & & Decreased & Decreased & \\
\hline Cold growth & Increased CspB & & Decreased & Decreased & & & & \\
\hline Antibiotic & $\mathrm{AG}^{r}, \beta \mathrm{L}^{r}$, Dapto $^{\mathrm{S}}$ & SXTr, & & & & $A G^{r}$ & & \\
\hline
\end{tabular}

$A G^{r}$, aminoglycoside resistant; $\beta L^{r}$, betalactam resistant; Daptos, daptomycin susceptible; ClpC, ATP-dependent protease C; ClpP, ATP-dependent protease P; CSP, capsular polysaccharide; CspB, cold shock protein B; FnBP, fibronectin binding protein; Hla, $\alpha$-toxin; HId, $\delta$-toxin; Ilv, Isoleucin, leucine, valine; Spa, Protein A; SXTr, sulfamethoxazole-trimethoprim resistant; sucD, succinyl-CoA synthetase subunit alpha; TCA, tricarboxylic acid cycle.

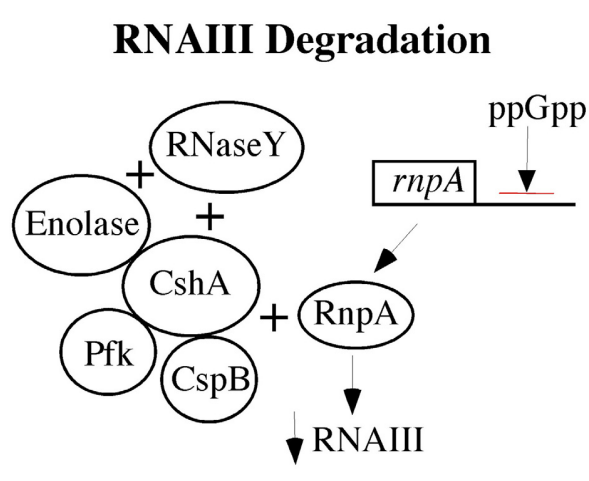

FIGURE 1 | The RNA degrasome is shown with respect to its impact on RNAIII as low levels of RNAIII are associated with persistent $\boldsymbol{S}$. aureus infections. An ATP-dependent $S$. aureus hellicase (CshA) is shown at the center with three proteins associated with it: Enolase, phosphofructokinase (Pfk), and cold shock protein B (CspB). Two RNase are shown, RNaseY and RnpA. RnpA has activity against RNAlll and its gene is negatively regulated by the alarmone, ppGpp.

variants. Mutation of a sRNA, $r s a A$, has been show to alter virulence and cause persistent infection (Romilly et al., 2014). RsaA binds to MgrA, a global transcriptional regulator, and attenuates the severity of infections while increasing biofilm formation. Hence, it contributes to chronic infections (Romilly et al., 2014).

The higher levels of RsaA seen in electron transport SCVs might help to explain the increased biofilm formation in these variants as RsaA represses the synthesis of MgrA thereby increasing biofilm (Seggewiss et al., 2006). Also, SCVs have been reported to be readily killed by phagocytes (Quie, 1969), and this is also true of strains over-expressing RsaA (Seggewiss et al., 2006). Hence, increased amounts of RsaA may be contributing to the SCV phenotype. (A more detailed review of sRNAs in S. aureus is in Felden et al. (2011).

\section{TOXIN-ANTITOXIN, MazEF, AND SCVs}

Another system that is involved in mRNA stability in S. aureus is the MazEF toxin-antitoxin system (Fu et al., 2007, 2009; Donegan and Cheung, 2009; Donegan et al., 2010; Figure 2). Toxinantitoxin systems are produced by many bacteria (Magnuson, 2007) and transcribed as an operon so that the toxin (MazF) is readily bond and neutralized by the antitoxin (MazE) (Fu et al., 2007, 2009; Donegan and Cheung, 2009; Donegan et al., 2010). MazE is an RNase that acts upon selected mRNAs. In S. aureus, MazE targets sigB, hla, spa mRNAs, but it avoids mRNAs from $\operatorname{rec} A, \operatorname{gyrB}$, sarA (Fu et al., 2007, 2009). In most bacteria, the MazEF system is regulated by feedback inhibition by its own gene products, but in $S$. aureus, the negative regulation comes via SigB (Donegan and Cheung, 2009). The mazEF promoter allows transcription of not only mazEF but also the sigB operon, and activation of the mazEF promoter is needed for full SigB activity.

High levels of free MazF result in slow growth (SCVs), decreased pigment, and persistence, which can be achieved by overexpression of MazF or increased activity of ATP-dependent 


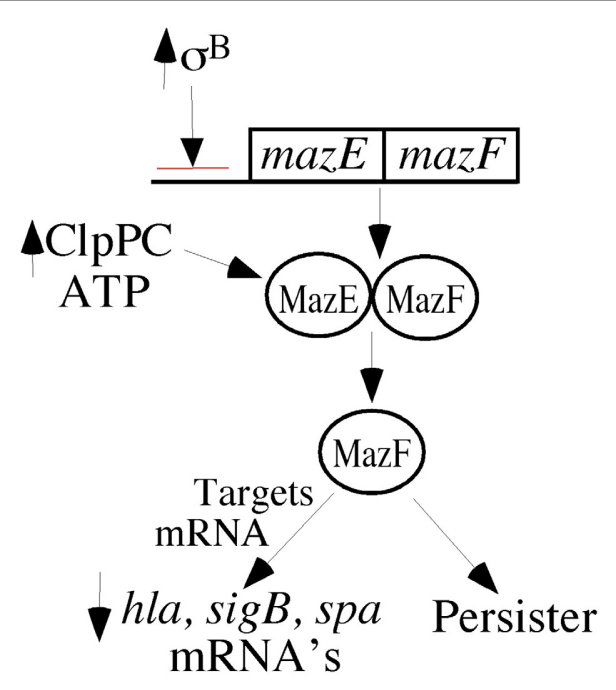

FIGURE 2 | MazEF are transcripbed as a pair of proteins, and their transcription is negatively regulated by SigmaB. MazE is an anti-toxin protein that binds to MazF, thereby preventing its activity. MazF has RNase activity, which targets selected mRNAs in S. aureus. The ATP-dependent protease pair, ClpPC, digests the MazE antitoxin, thereby releasing MazF. Increased MazF has been associated with persistence.

protease system, ClpCP, which proteolytically targets the antitoxin, MazE (Fu et al., 2007, 2009; Donegan and Cheung, 2009; Donegan et al., 2010). This is compatible with the concept that toxin-antitoxin systems are involved in persistence (Magnuson, 2007). On the other hand, the low levels of ATP and ClpP and the higher levels $\sigma^{\mathrm{B}}$ in electron transport SCVs suggest that the MazEF system is probably not contributing to persistence in electron transport SCVs as electron transport SCVs have high levels of SigB (Mitchell et al., 2013).

\section{MUTATION IN rpLF AND SCVs}

The mutations in $r l f$, which encodes ribosomal protein L6, cause SCVs that have decreased pigmentation, like electron transport type of SCVs, and that allow for persistence (Norström et al., 2007; Lannergård et al., 2011). L6 is required for efficient protein synthesis, thus it is not surprising that cells with defective L6 would grow slowly. Rfl is also called FusE, which comes from the fact that these mutants are resistant to fusidic acid because of changes in ribosomal protein L6. In addition, $r f l$ mutation in E. coli results in increased membrane fluidity and aminoglycoside resistance (Bosl and Böck, 1981), which is also seen with electron transport deficient SCVs. Aminoglycosides can also select for simultaneous fusidic acid resistance (Norström et al., 2007; Lannergård et al., 2011). Of note, in S. aureus, L6 is reduced in hemB mutants and under anaerobic growth (Fuchs et al., 2007; Kriegeskorte et al., 2011), suggesting that part of the phenotype seen in electron transport SCVs may relate to changes in L6. Little more in terms of the phenotype is known except that many, but not all of the $\mathrm{rfl}$ mutants, also carry mutations in hem or men ( $g e r C)$. These would be double SCVs as a mutation in rlf alone is sufficient to produce an SCV phenotype. Again, we see the ribosome, hence RNA, being involved in persistence and the SCV phenotype.

\section{STRINGENT RESPONSE, PERSISTENCE, AND SCVs}

When investigating a $S$. aureus strain from a patient with persistent and recurrent infection, a constitutively active mutation in $r s h$, a RelA homolog in S. aureus, was recovered (Gao et al., 2010). The mutation resulted defective ppGpp hypdrolase activity, thereby giving high levels of ppGpp and a persistent stringent response (Gao et al., 2010). Resistance to linezolid was also found. As ppGpp is the effector molecule in the stringent response (Geiger et al., 2012, 2014; Geiger and Wolz, 2014), which limits protein synthesis, it is not surprising that there is a growth defect, hence small colonies, i.e., an SCV (Geiger et al., 2012, 2014; Geiger and Wolz, 2014). Mutations in rsh result in reduced virulence (Geiger et al., 2012, 2014; Geiger and Wolz, 2014). Aside from the small colony size, the $r$ sh mutant does not resemble the electron transport SCVs because $r s h$ mutants show increased RNAIII and all of the characteristics seen when the Agr regulon is active.

\section{ARGININE DEIMINASE AND PERSISTENCE}

In electron transport deficient SCVs, the agrABDC pathway, which is responsible for arginine deiminase activity, is markedly increased aerobically, whereas in the parent strains it is expressed only anaerobically (Kohler et al., 2003, 2008; Seggewiss et al., 2006; Makhlin et al., 2007). The anaerobic regulator of this operon, $\operatorname{ArgR}$, is also significantly increased in the hemB mutant (Seggewiss et al., 2006), and it also regulates other normally anaerobic genes (Makhlin et al., 2007). This is of interest because the arginine catabolic mobile element (ACME) in USA300 is found along with the SCCmec element and is expressed aerobically whereas the other arginine deiminase operon are expressed only anaerobically (Thurlow et al., 2013). S. aureus USA300 is the hypervirulent epidemic strain in the USA. Arginine metabolism by ACME allows $S$. aureus to survive on the acidic environment of skin and within skin abscesses because it neutralizes acid with ammonia released during arginine catabolism (Thurlow et al., 2013). This provides a mechanism for the epidemiological observation that USA300 has an increased propensity to colonize the acidic environment of the skin chronically, which can be considered a type of persistence (Miko et al., 2012). Within ACME is another gene, spe $G$, which encodes, spermidine acetyltransferase, a polyamine-resistance enzyme, thereby allowing $S$. aureus to survive spermidine exposure on the skin and in abscesses (Thurlow et al., 2013). Spermidine is otherwise a toxic product of arginine metabolism (Thurlow et al., 2013). The elucidation of the role of ACME in USA300 helps in the understanding how electron transport SCVs are able to survive for longer periods of time than other strains of $S$. aureus by using similar metabolic mechanisms.

\section{METABOLISM AND ANTIBIOTIC-PERSISTERS}

A large literature is available linking slow growth and resistance to antibiotics (Wood et al., 2013), and this is also found in S. aureus (Lechner et al., 2012). Discussing this type of persistence is beyond the scope of this review; however, one specific gene will be covered as it has a special link between phosphate metabolism and slow growth, hence an SCV, specifically phoU. 
PhoU is a global negative regulator that regulates genes involved in central carbon metabolism and cytochrome expression, thus it provides strong links to the electron transport type of SCVs ( $\mathrm{Li}$ and Zhang, 2007; Gardner et al., 2014). In S. aureus, phoU can be found to be important for resistance to cationic antimicrobial peptides (Overton et al., 2011), which may relate to expression of the $d l t$ and $s n o D$ operons that have been related to cationic antimicrobial peptide resistance (Peschel et al., 1999; Bayer et al., 2006). Both the $d l t$ and snoD operons are regulated by PhoU. We have found that the electron transport type of SCVs are also persist in the presence of antibiotics and more resistant to cationic peptides (Koo et al., 1996; Gläser et al., 2014). Studies of S. aureus phoU mutants ability to persist within the host are not available, but one would surmise that this may be the case.

\section{CONCLUSIONS}

Persistence and metabolism are intimately intertwined (Kahl, 2014), and it can be achieved via altering many pathways. Slow growth is common theme for persisters. The electron transport deficient SCVs show changes in many of the other pathways used for persistence, hence, the studies of these SCVs have a broader relevance for persistence. Of interest, altered RNA processing is suggested in the electron transport SCVs by the exceptionally low levels of RNAIII. A number of other persister types also show changes in RNA metabolism either directly through the RNA degrasome or at the ribosome. While the loss of agr is associated strongly with persistent and chronic infections, it is not associated with a growth defect. Naturally, there is a clear RNA effect in $a g r$ mutants in that the effector molecule, RNAIII, is not produced. Thus, there are many connections between the SCV phenotype and altered mRNA levels. There are also a number of shared phenotypic characteristics PhoU mediated changes and SCVs, but direct connections have yet to be studied. Finally, a growth defect is seen with high ppGpp levels, but these strains need to be tested for persistence.

\section{REFERENCES}

Abdallah, M., Benoliel, C., Drider, D., Dhulster, P., and Chihib, N. E. (2014). Biofilm formation and persistence on abiotic surfaces in the context of food and medical environments. Arch. Microbiol. doi: 10.1007/s00203-014-0983-1

Abu-Qatousch, L. F., Chinni, S. V., Seggewiss, J., Proctor, R. A., Brosius, J., Rozhdestvensky, T. S., et al. (2010). Identification of differentially expressed small non-protein-coding RNAs in Staphylococcus aureus displaying both the normal and the small-colony variant phenotype. J. Mol. Med. 88, 565-575. doi: 10.1007/s00109-010-0597-2

Amato, S. M., Fazen, C. H., Henry, T. C., Mok, W. W., Orman, M. A., Sandvik, E. L., et al. (2014). The role of metabolism in bacterial persistence. Front. Microbiol. 5:70. doi: 10.3389/fmicb.2014.00070

Balwit, J. M., van Langevelde, P., Vann, J. M., and Proctor, R. A. (1994). Gentamicin-resistant menadione and hemin auxotrophic Staphylococcus aureus persist within cultured endothelial cells. J. Infect. Dis. 170, 1033-1037. doi: 10.1093/infdis/170.4.1033

Bayer, A. S., McNamara, P., Yeaman, M. R., Lucindo, N., Jones, T., Cheung, A. L., et al. (2006). Transposon disruption of the complex I NADH oxidoreductase gene (snoD) in Staphylococcus aureus is associated with reduced susceptibility to the microbicidal activity of thrombin-induced platelet microbicidal protein. J. Bacteriol. 188, 211-222. doi: 10.1128/JB.188.1.211-222.2006

Beenken, K. E., Dunman, P. M., McAleese, F., Macapagal, D., Murphy, E., Projan, S. J., et al. (2004). Global gene expression in Staphylococcus aureus biofilms. J. Bacteriol. 186, 4665-4684. doi: 10.1128/JB.186.14.46654684.2004
Bohn, C., Rigoulay, C., Chabelskaya, S., Sharma, C. M., Marchais, A., Skorski, P., et al. (2010). Experimental discovery of small RNAs in Staphylococcus aureus reveals a riboregulator of central metabolism. Nucleic Acids Res. 38, 6620-6636. doi: 10.1093/nar/gkq462

Bosl, A., and Böck, A. (1981). Ribosomal mutation in Escherichia coli affecting membrane stability. Mol. Gen. Genet. 182, 358-360. doi: 10.1007/BF00269684

Chatterjee, I., Becker, P., Grundmeier, M., Bischoff, M., Somerville, G. A., Peters, G., et al. (2005). Staphylococcus aureus ClpC is required for stress resistance, aconitase activity, growth recovery, and death. J. Bacteriol. 187, 4488-4496. doi: 10.1128/JB.187.13.4488-4496.2005

Chatterjee, I., Herrmann, M., Proctor, R. A., Peters, G., and Kahl, B. C. (2007). Enhanced post-stationary-phase survival of a clinical thymidine-dependent small-colony variant of Staphylococcus aureus results from lack of a functional tricarboxylic acid cycle. J. Bacteriol. 189, 2936-2940. doi: 10.1128/JB.01444-06

Chatterjee, I., Kriegeskorte, A., Fischer, A., Deiwick, S., Theimann, N., Proctor, R. A., et al. (2008). In vivo mutations of thymidylate synthase (encoded by thyA) are responsible for thymidine dependency in clinical small-colony variants of Staphylococcus aureus. J. Bacteriol. 190, 834-842. doi: 10.1128/JB.00912-07

Clements, M. O., Watson, S. P., Poole, R. K., and Foster, S. J. (1999). CtaA of Staphylococcus aureus is required for starvation survival, recovery, and cytochrome biosynthesis. J. Bacteriol. 181, 501-507.

Donegan, N. P., and Cheung, A. L. (2009). Regulation of the mazEF toxin-antitoxin module in Staphylococcus aureus and its impact on $\operatorname{sigB}$ expression. J. Bacteriol. 191, 2795-2805. doi: 10.1128/JB.01713-08

Donegan, N. P., Thompson, E. T., Fu, Z., and Cheung, A. L. (2010). Proteolytic regulation of toxin-antitoxin systems by ClpPC in Staphylococcus aureus. J. Bacteriol. 192, 1416-1422. doi: 10.1128/JB.00233-09

Duval, B. D., Mathew, A., Satola, S. W., and Shafer, W. M. (2010). Altered growth, pigmentation, and antimicrobial susceptibility properties of Staphylococcus aureus due to loss of the major cold shock gene $\operatorname{cspB}$. Antimicrob. Agents Chemother. 54, 2283-2290. doi: 10.1128/AAC.01786-09

Edwards, A. M. (2012). Phenotype switching is a natural consequence of Staphylococcus aureus replication. J. Bacteriol. 194, 5404-5412. doi: 10.1128/JB.00948-12

Felden, B., Vandenesch, F., Bouloc, P., and Romby, P. (2011). The Staphylococcus aureus RNome and its commitment to virulence. PLoS Pathog. 7:e1002006. doi: 10.1371/journal.ppat.1002006

Fu, Z., Donegan, N. P., Memmi, G., and Cheung, A. L. (2007). Characterization of MazFSa, an endoribonuclease from Staphylococcus aureus. J. Bacteriol. 191, 2051-2059. doi: 10.1128/JB.00907-08

Fu, Z., Tamber, S., Memmi, G., Donegan, N. P., and Cheung, A. L. (2009). Overexpression of MazFsa in Staphylococcus aureus induces bacteriostasis by selectively targeting mRNAs for cleavage. J. Bacteriol. 191, 2051-2059. doi: 10.1128/JB.00907-08

Fuchs, S., Pané-Farr,é, J., Kohler, C., Hecker, M., and Engelmann, S. (2007). Anaerobic gene expression in Staphylococcus aureus. J. Bacteriol. 189, 4275-4289. doi: 10.1128/JB.00081-07

Gao, W., Chua, K., Davies, J. K., Newton, H. J., Seemann, T., Harrison, P. F., et al. (2010). Two novel point mutations in clinical Staphylococcus aureus reduce linezolid susceptibility and switch on the stringent response to promote persistent infection. PLoS Pathog. 6:e1000944. doi: 10.1371/journal.ppat.1000944

Garcia, L., Lemaire, S., Kahl, B., Becker, K., Proctor, R., Denis, O., et al. (2013). Antibiotic activity against small colony variants of Staphylococcus aureus: review of in vitro, animal, and clinical data. J. Antimicrob. Chemother. 68, 1455-1465. doi: 10.1093/jac/dkt072

Gardner, S. G., Johns, K. D., Tanner, R., and McCleary, W. R. (2014). The PhoU protein from Escherichia coli interacts with PhoR, PstB, and metals to form a phosphate-signaling complex at the membrane. J. Bacteriol. 196, 1741-1752. doi: 10.1128/JB.00029-14

Geiger, T., Francois, P., Liebeke, M., Fraunholz, M., Goerke, C., Krismer, B., et al. (2012). The stringent response of Staphylococcus aureus and its impact on survival after phagocytosis through the induction of intracellular PSMs expression. PLoS Pathog. 8:e1003016. doi: 10.1371/journal.ppat.1003016

Geiger, T., Kästle, B., Gratani, F. L., Goerke, C., and Wolz, C. (2014). Two small (p)ppGpp synthases in Staphylococcus aureus mediate tolerance against cell envelope stress conditions. J. Bacteriol. 196, 894-902. doi: 10.1128/JB.01201-13

Geiger, T., and Wolz, C. (2014). Intersection of the stringent response and the CodY regulon in low GC Gram-positive bacteria. Int. J. Med. Microbiol. 304, 150-155. doi: 10.1016/j.ijmm.2013.11.013 
Geissmann, T., Chevalier, C., Cros, M.-J., Boisset, S., Fechter, P., Noirot, C., et al. (2009). A search for small noncoding RNAs in Staphylococcus aureus reveals a conserved sequence motif for regulation. Nuclic Acid Res. 37, 7239-7257. doi: 10.1093/nar/gkp668

Gläser, R., Becker, K., von Eiff, C., Meyer-Hoffert, U., and Harder, J. (2014). Decreased susceptibility of Staphylococcus aureus small-colony variants toward human antimicrobial peptides. J. Invest. Dermatol. doi: 10.1038/jid.2014.176

Guillet, J., Hallier, M., and Felden, B. (2013). Emerging functions for the Staphylococcus aureus RNome. PLoS Pathog. 9:31003767. doi: 10.1371/journal.ppat. 1003767

Hirschhausen, N., Block, D., Bianconi, I., Bragonzi, A., Birtel, J., Lee, J. C., et al. (2013). Extended Staphylococcus aureus persistence in cystic fibrosis is associated with bacterial adaptation. Int. J. Med. Microbiol. 303, 685-692. doi: 10.1016/j.ijmm.2013.09.012

Horst, S. A., Hoerr, V., Beineke, A., Kreis, C., Tuchscherr, L., Kalinka, J., et al. (2012). A novel mouse model of Staphylococcus aureus chronic osteomyelitis that closely mimics the human infection: an integrated view of disease pathogenesis. Am. J. Pathol. 181, 1206-1214. doi: 10.1016/j.ajpath.2012.07.005

Hunger, K., Beckering, C. L., Wiegeshoff, F., Graumann, P. L., and Marahiel, M. A. (2006). Cold-induced putative DEAD box RNA helicases CshA and CshB are essential for cold adaptation and interact with cold shock protein B in Bacillus subtilis. J. Bacteriol. 188, 240-248. doi: 10.1128/JB.188.1.240-248.2006

Kahl, B. C. (2014). Small colony variants (SCVs) of Staphylococcus aureus A bacterial survival strategy. Infect. Genet. Evol. 21, 515-521. doi: 10.1016/j.meegid.2013.05.016

Kahl, B. C., Belling, G., Becker, P., Chatterjee, I., Wardecki, K., Hilgert, K., et al. (2005). Thymidine-dependent Staphylococcus aureus small-colony variants are associated with extensive alterations in regulator and virulence gene expression profiles. Infect. Immun. 73, 4119-4126. doi: 10.1128/IAI.73.7.4119-4126.2005

Kohler, C., von Eiff, C., Liebeke, M., McNamara, P. J., Lalk, M., Proctor, R. A., et al. (2008). A defect in menadione biosynthesis induces global changes in gene expression in Staphylococcus aureus: comparisons to the hemB mutant. J. Bacteriol. 190, 6351-6364. doi: 10.1128/JB.00505-08

Kohler, C., von Eiff, C., Peters, G., Proctor, R. A., Hecker, M., and Engelmann, S. (2003). Physiological characterization of a heme-deficient mutant of Staphylococcus aureus by a proteomic approach. J. Bacteriol. 185, 6928-6937. doi: 10.1128/JB.185.23.6928-6937.2003

Koo, S. P., Bayer, A. S., Sahl, H. G., Proctor, R. A., and Yeaman, M. R. (1996). Staphylocidal action of thrombin-induced platelet microbicidal protein is not solely dependent on transmembrane potential. Infect. Immun. 64, 1070-1074.

Kriegeskorte, A., König, S., Sander, G., Pirkl, A., Mahabir, E., Proctor, R. A., et al. (2011). Small colony variants of Staphylococcus aureus reveal distinct protein profiles. Proteomics 11, 2476-2490. doi: 10.1002/pmic.201000796

Lannergård, J., Cao, S., Norström, T., Delgado, A., Gustafson, J. E., and Hughes, D. (2011). Genetic complexity of fusidic acid-resistant small colony variants (SCV) in Staphylococcus aureus. PLoS ONE 6:e28366. doi: 10.1371/journal.pone.0028366

Lechner, S., Lewis, K., and Bertram, R. (2012). Staphylococcus aureus persisters tolerant to bactericidal antibiotics. J. Mol. Microbiol. Biotechnol. 22, 235-244. doi: $10.1159 / 000342449$

Li, Y., and Zhang, Y. (2007). PhoU is a persistence switch involved in persister formation and tolerance to multiple antibiotics and stresses in Escherichia coli. Antimicrob. Agents Chemother. 51, 2092-2029. doi: 10.1128/AAC.00052-07

Lowy, F. D. (1998). Staphylococcus aureus infections. N. Engl. J. Med. 339, 520-532. doi: 10.1056/NEJM199808203390806

Magnuson, R. D. (2007). Hypothetical functions of toxin-antitoxin systems. J. Bacteriol. 189, 6089-6092. doi: 10.1128/JB.00958-07

Makhlin, J., Kofman, T., Borovok, I., Kohler, C., Engelmann, S., Cohen, G., et al. (2007). Staphylococcus aureus ArcR controls expression of the arginine deiminase operon. J. Bacteriol. 189, 5976-5986. doi: 10.1128/JB.00592-07

Marincola, G., Schäfer, T., Behler, J., Bernhardt, J., Ohlsen, K., Goerke, C., et al. (2012). RNase Y of Staphylococcus aureus and its role in the activation of virulence genes. Mol. Microbiol. 85, 817-832 doi: 10.1111/j.1365-2958.2012.08144.x

Massey, R. C., Buckling, A., and Peacock, S. J. (2001). Phenotypic switching of antibiotic resistance circumvents permanent costs in Staphylococcus aureus. Curr. Biol. 11, 1810-1814. doi: 10.1016/S0960-9822(01)00507-3

McNamara, P. J., Syverson, R. E., Milligan-Myhre, K., Frolova, O., Schroeder, S., Kidder, J., et al. (2009). Surfactants, aromatic and isoprenoid compounds, and fatty acid biosynthesis inhibitors suppress Staphylococcus aureus production of toxic shock syndrome toxin 1. Antimicrob. Agents Chemother. 53, 1898-1906. doi: 10.1128/AAC.01293-08

Miko, B. A., Uhlemann, A. C., Gelman, A., Lee, C. J., Hafer, C. A., Sullivan, S. B., et al. (2012). High prevalence of colonization with Staphylococcus aureus clone USA300 at multiple body sites among sexually transmitted diseases clinic patients: an unrecognized reservoir. Microbes Infect. 14, 1040-1043. doi: 10.1016/j.micinf.2012.06.004

Mitchell, G., Fugère, A., Pépin Gaudreau, K., Brouillette, E., Frost, E. H., Cantin, A. M., et al. (2013). SigB is a dominant regulator of virulence in Staphylococcus aureus small-colony variants. PLoS ONE 8:e65018. doi: 10.1371/journal.pone.0065018

Morikawa, K., Ohniwa, R. L., Ohta, T., Tanaka, Y., Takeyasu, K., and Msadek, T. (2010). Adaptation beyond the stress response: cell structure dynamics and population heterogeneity in Staphylococcus aureus. Microbes Environ. 25, 75-82. doi: 10.1264/jsme2.ME10116

Norström, T., Lannergård, J., and Hughes, D. (2007). Genetic and phenotypic identification of fusidic acid-resistant mutants with the small-colony-variant phenotype in Staphylococcus aureus. Antimicrob. Agents Chemother. 51, 4438-4446. doi: 10.1128/AAC.00328-07

Novick, R. P., and Geisinger, E. (2008). Quorum sensing in staphylococci. Annu. Rev. Genet. 42, 541-564. doi: 10.1146/annurev.genet.42.110807.091640

Oun, S., Redder, P., Didier, J. P., François, P., Corvaglia, A. R., Buttazzoni, E., et al. (2013). The CshA DEAD-box RNA helicase is important for quorum sensing control in Staphylococcus aureus. RNA Biol. 10, 157-165. doi: 10.4161/rna.22899

Overton, I. M., Graham, S., Gould, K. A., Hinds, J., Botting, C. H., Shirran, S., et al. (2011). Global network analysis of drug tolerance, mode of action and virulence in methicillin-resistant S. aureus. BMC Syst. Biol. 5:68. doi: 10.1186/1752-0509-5-68

Peschel, A., Otto, M., Jack, R. W., Kalbacher, H., Jung, G., and Götz, F. (1999). Inactivation of the dlt operon in Staphylococcus aureus confers sensitivity to defensins, protegrins, and other antimicrobial peptides. J. Biol. Chem. 274, 8405-8410. doi: 10.1074/jbc.274.13.8405

Pragman, A. A., Yarwood, J. M., Tripp, T. J., and Schlievert, P. M. (2004). Characterization of virulence factor regulation by SrrAB, a twocomponent system in Staphylococcus aureus. J. Bacteriol. 186, 2430-2438. doi: 10.1128/JB.186.8.2430-2438.2004

Proctor, R. A., van Lagevelde, P., Kristjansson, M., Maslow, J., and Arbeit, R. D. (1995). Persistent and relapsing infections associated with small-colony variants of Staphylococcus aureus. Clin. Infect. Dis. 20, 95-102, doi: 10.1093/clinids/20.1.95

Proctor, R. A., von Eiff, C., Kahl, B. C., Becker, K., McNamara, P., Herrmann, M., et al. (2006). Small colony variants: a pathogenic form of bacteria that facilitates persistent and recurrent infections. Nat. Microb. Rev. 4, 295-305. doi: $10.1038 /$ nrmicro1384

Quie, P. G. (1969). Microcolonies (G-variants) of Staphylococcus aureus. Yale J. Biol. Med. 41, 394-403.

Redder, P., and Linder, P. (2012). DEAD-box RNA helicases in gram-positive RNA decay. Methods Enzymol. 511, 369-383. doi: 10.1016/B978-0-12-3965462.00017-6

Romilly, C., Lays, C., Tomasini, A., Caldelari, I., Benito, Y., Hammann, P., et al. (2014). A non-coding RNA promotes bacterial persistence and decreases virulence by regulating a regulator in Staphylococcus aureus. PLoS Pathog. 10:e1003979. doi: 10.1371/journal.ppat.1003979

Rothfork, J. M., Dessus-Babus, S., Van Wamel, W. J., Cheung, A. L., and Gresham, H. D. (2003). Fibrinogen depletion attenuates Staphylococcus aureus infection by preventing density-dependent virulence gene up-regulation. J. Immunol. 171, 5389-5395. doi: 10.4049/jimmunol.171.10.5389

Roux, C. M., DeMuth, J. P., and Dunman, P. M. (2011). Characterization of components of the Staphylococcus aureus mRNA degradosome holoenzyme-like complex. J. Bacteriol. 193, 5520-5526. doi: 10.1128/JB.05485-11

Samuelsen, Ø., Haukland, H. H., Kahl, B., von Eiff, C., Proctor, R. A., Ulvatne, H., et al. (2005). Staphylococcus aureus small colony variants are resistant to the antimicrobial peptide lactoferricin B. J. Antimicrobial. Chemother. 56, 1126-1129. doi: 10.1093/jac/dki385

Sarafian, S. K., and Morse, S. A. (1987). Environmental factors affecting toxic shock syndrome toxin-1 (TSST-1) synthesis. J. Med. Microbiol. 24, 75-81. doi: 10.1099/00222615-24-1-75

Schwan, W. R., Langhorne, M. H., Ritchie, H. D., and Stover, C. K. (2003). Loss of hemolysin expression in Staphylococcus aureus agr mutants correlates with 
selective survival during mixed infections in murine abscesses and wounds. FEMS Immunol. Med. Microbiol. 38, 23-28. doi: 10.1016/S0928-8244(03) 00098-1

Seggewiss, J., Becker, K., Kotte, O., Eisenacher, M., Yazdi, M. R., Fischer, A., et al. (2006). Reporter metabolite analysis of transcriptional profiles of a Staphylococcus aureus strain with normal phenotype and its isogenic hemB mutant displaying the small-colony-variant phenotype. J. Bacteriol. 188, 7765-7777. doi: 10.1128/JB.00774-06

Sendi, P., and Proctor, R. A. (2009). Staphylococcus aureus as an intracellular pathogen: the role of small colony variants. Trends Microbiol. 17, 54-55. doi: 10.1016/j.tim.2008.11.004

Somerville, G. A., Chaussee, M. S., Morgan, C. I., Fitzgerald, J. R., Dorward, D. W., Reitzer, L. J., et al. (2002). Staphylococcus aureus aconitase inactivation unexpectedly inhibits post-exponential-phase growth and enhances stationaryphase survival. Infect. Immun. 70, 6373-6382. doi: 10.1128/IAI.70.11.63736382.2002

Thurlow, L. R., Joshi, G. S., Clark, J. R., Spontak, J. S., Neely, C. J., Maile, R., et al. (2013). Functional modularity of the arginine catabolic mobile element contributes to the success of USA300 methicillin-resistant Staphylococcus aureus. Cell Host Microbe 13, 100-107. doi: 10.1016/j.chom.2012.11.012

Tuchscherr, L., Heitmann, V., Hussain, M., Viemann, D., Roth, J., von Eiff, C., et al. (2010). Staphylococcus aureus small-colony variants are adapted phenotypes for intracellular persistence. J. Infect. Dis. 202, 1031-1040. doi: 10.1086/ 656047

Tuchscherr, L., Medina, E., Hussain, M., Völker, W., Heitmann, V., Niemann, S., et al. (2011). Staphylococcus aureus phenotype switching: an effective bacterial strategy to escape host immune response and establish a chronic infection. EMBO Mol. Med. 3, 129 - 141. doi: 10.1002/emmm.201000115

Vaudaux, P., Francois, P., Bisognano, C., Kelley, W. L., Lew, D. P., Schrenzel, J., et al. (2002). Increased expression of clumping factor and fibronectin-binding proteins by hemB mutants of Staphylococcus aureus expressing small colony variant phenotypes. Infect. Immun. 70, 5428-5437. doi: 10.1128/IAI.70.10.54285437.2002

Vesga, O., Groeschel, M. C., Otten, M. F., Brar, D. W., Vann, J. M., and Proctor, R. A. (1996). Staphylococcus aureus small colony variants are induced by the endothelial cell intracellular milieu. J. Infect. Dis. 173, 739-742. doi: 10.1093/infdis/173.3.739

Werth, N., Beerlage, C., Rosenberger, C., Yazdi, A. S., Edelmann, M., Amr, A., et al. (2010). Activation of hypoxia inducible factor 1 is a general phenomenon in infections with human pathogens. PLOS ONE 5:e11576. doi: 10.1371/journal.pone.0011576

Wood, T. K., Knabel, S. J., and Kwan, B. W. (2013). Bacterial persister cell formation and dormancy. Appl Environ Microbiol 79, 7116-7121. doi: 10.1128/AEM.02636-13

Wright, J. S. 3rd, Jin, R., and Novick, R. P. (2005). Transient interference with staphylococcal quorum sensing blocks abscess formation. Proc. Natl. Acad. Sci. U.S.A. 102, 1691-1696. doi: 10.1073/pnas.0407661102

Xue, T., Zhang, X., Sun, H., and Sun, B. (2014). ArtR, a novel sRNA of Staphylococc us aureus, regulates $\alpha$-toxin expression by targeting the 5'UTR of sarT mRNA. Med. Microbiol. Immunol. 203, 1-12. doi: 10.1007/s00430-013-0307-0

Yarwood, J. M., and Schlievert, P. M. (2003). Quorum sensing in Staphylococcus infections. J. Clin. Invest. 112, 1620-1625. doi: 10.1172/JCI200320442

Conflict of Interest Statement: The authors declare that the research was conducted in the absence of any commercial or financial relationships that could be construed as a potential conflict of interest.

Received: 16 May 2014; accepted: 07 July 2014; published online: 28 July 2014. Citation: Proctor RA, Kriegeskorte A, Kahl BC, Becker K, Löffler B and Peters G (2014) Staphylococcus aureus Small Colony Variants (SCVs): a road map for the metabolic pathways involved in persistent infections. Front. Cell. Infect. Microbiol. 4:99. doi: $10.3389 /$ fcimb. $^{2014.00099}$

This article was submitted to the journal Frontiers in Cellular and Infection Microbiology.

Copyright (C) 2014 Proctor, Kriegeskorte, Kahl, Becker, Löfler and Peters. This is an open-access article distributed under the terms of the Creative Commons Attribution License (CC BY). The use, distribution or reproduction in other forums is permitted, provided the original author(s) or licensor are credited and that the original publication in this journal is cited, in accordance with accepted academic practice. No use, distribution or reproduction is permitted which does not comply with these terms. 